\title{
Case Study of an Old Woman With Complain of Recurrent Abdominal
}

\section{Pain}

\author{
Ahmad Hormati, ${ }^{1}$ and Pezhman Alavinejad ${ }^{2, *}$ \\ ${ }^{1}$ Assistant Professor of Gastroenterology and Hepatology, Qom Digestive Disease Research Center, Qom University of Medical Sciences, Qom, IR Iran \\ ${ }^{2}$ Assistant Professor of Gastroenterology, Research Center for Infectious Diseases of Digestive System, Ahvaz Jundishapur University of Medical Sciences, Ahvaz, IR Iran \\ "Corresponding author: Pezhman Alavinejad, Assistant Professor of Gastroenterology, Research Center for Infectious Diseases of Digestive System, Ahvaz Jundishapur \\ University of Medical Sciences, Ahvaz, IR Iran. E-mail: pezhmanalavinejad@gmail.com
}

Received 2016 April 15; Revised 2016 August 28; Accepted 2016 August 29.

\begin{abstract}
Introduction: The evaluation of acute abdominal pain must be efficient to prevent any delay in the treatment of patients, who are seriously ill, and over treatment of patients with self-limited disorders. One of the uncommon reasons that should be considered in the differential diagnosis of acute abdominal pain, especially among elderly patients, who are under anti-coagulation therapy, is abdominal wall hematoma.

Case Presentation: The case was a 60-year-old female with abdominal pain in the left upper and lower quadrant, which was followed by a series of coughs. She was receiving subcutaneous heparin, which was then changed to warfarin. The patient also had an episode of similar pain, one month ago and was diagnosed as abdominal hematoma based on ultrasonography report. An abdominal CT scan showed a mass lesion and the patient was diagnosed as acute on chronic abdominal wall hematoma.

Conclusions: Anterior abdominal wall hematoma should be considered in the differential diagnosis of acute abdominal pain among elderly patients, especially those who are under anti-coagulation therapy. Conservative treatment is conceivable in most cases and early diagnosis is compulsory to avoid morbidity or unnecessary surgery.
\end{abstract}

Keywords: Abdominal Pain, Hematoma, Elderly Patients

\section{Introduction}

Acute abdominal pain is a common complaint that brings patients to emergency departments. Approximately half of these patients have nonspecific findings and only a small proportion of patients have a life-threatening disease. Therefore, the evaluation of acute abdominal pain must be efficient and lead to an accurate diagnosis early in the presentation to prevent any delay in the treatment of patients, who are seriously ill and over treatment of patients with self-limited disorders (1). The information collected and a careful examination of the patient is more important than formal competence (2). One of the uncommon reasons that should be considered in the differential diagnosis of acute abdominal pain, especially among elderly patients, who are under anti- coagulation therapy, is abdominal wall hematoma (3). These hematomas are uncommon, but could result in a life-threatening condition. Risk factors include older age, female gender, systemic anticoagulation, abdominal wall trauma, pregnancy, and impaired renal function (4). In this case report, we briefly describe an old female patient, who presented acute abdominal pain due to chronic abdominal wall hematoma.

\section{Case Presentation}

A 60-year-old female patient presented abdominal pain in the Left Upper and Lower Quadrant (LUQ and LLQ), which was followed by a series of coughs. During the admission, her vital signs were stable and included: temperature of $36.8^{\circ} \mathrm{C}$, pulse of 72 beats/minute, respiratory rate of 20 breaths/minute, blood pressure of 134/71 $\mathrm{mmHg}$ and $\mathrm{O}_{2}$ saturation of $94 \%$. On physical examination the patient had tenderness and inflation in LUQ and LLQ, which became more sensitive upon contraction of abdominal muscles. She also had 3+ pitting edema and purpura on her lower extremity but the size of her legs was equal. She did not have lymphadenopathy. Laboratory results revealed: Hemoglobin $(\mathrm{Hb})=10.9$, mean corpuscular volume $(\mathrm{MCV})$ $=83.1$, whole blood count $(\mathrm{WBC}) 11300$, Erythrocyte sedimentary rate (ESR 32), C-reactive protein (CRP) ++ with normal partial Thromboplastin time (PTT) and Prothrombin time (PT).

On past medical history, she had diabetes Mellitus, high blood pressure, and congestive heart failure since 20 years ago, chronic renal failure, which evolved to end stage renal disease (ESRD) since three months and handled by hemodialysis, and also a history of thyroidectomy surgery 30 years ago. She was receiving subcutaneous hep- 


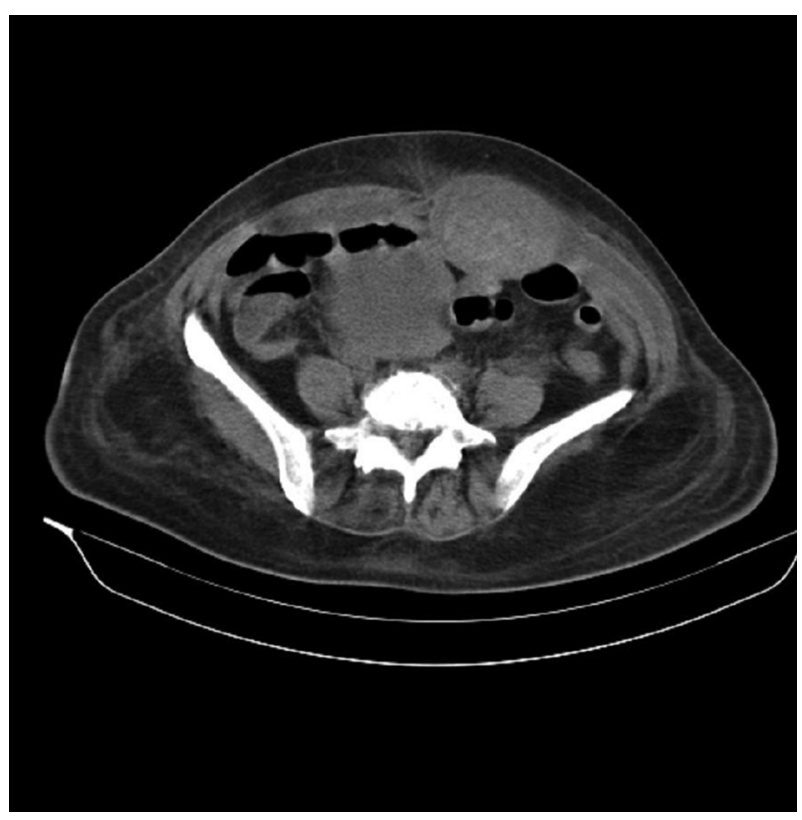

Figure 1. Abdominal Computerized Tomography Scan of Abdominal Mass Like Lesion Due to Hematoma

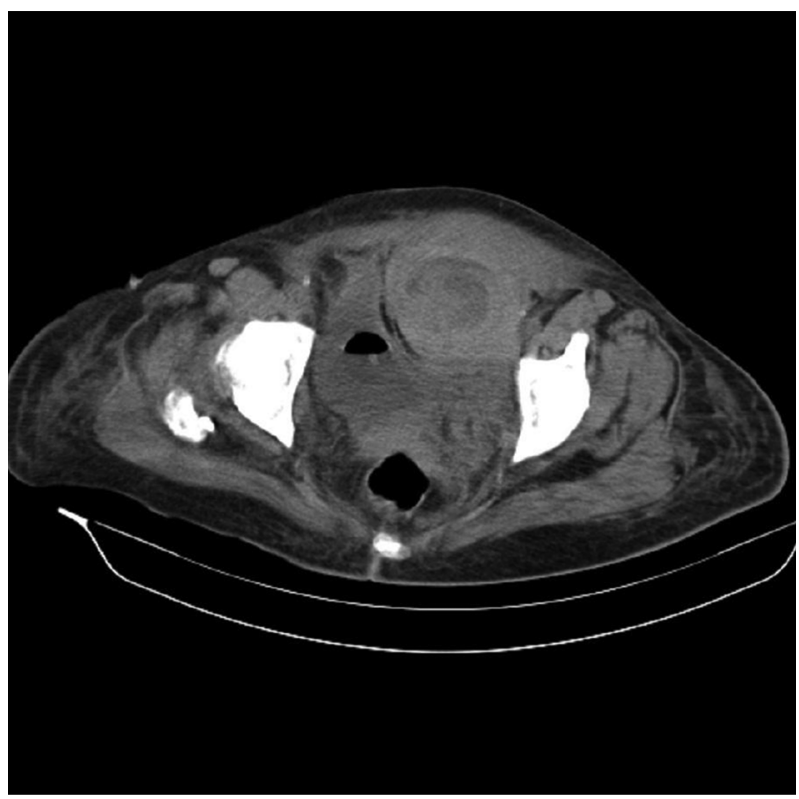

Figure 2. Abdominal Computerized Tomography Scan Showing Acute Chronic Abdominal Wall Hematoma

arin 5000 IU BID, which then changed to warfarin $5 \mathrm{mg}$ daily during the last six months. An abdominal computerized tomography (CT) scan was requested and her CT scan showed a mass lesion (Figures 1 and 2).

\section{Discussion}

This patient had an episode of similar pain one month ago, which was diagnosed as abdominal hematoma based on ultrasonography report. The hematoma in that session had a $2-\mathrm{cm}$ diameter and the current CT revealed enlargement of the previous lesion. Therefore, she was diagnosed with acute on chronic abdominal wall hematoma based on CT findings about the location and enhancement of lesion. The patients with ESRD are susceptible to hemostatic disorders due to bleeding tendency and platelet dysfunction. Hemorrhage can occur at retro-peritoneum, intracranial or other areas such as cutaneous, mucosal, or even serosal surfaces (5).

Several risk factors have been associated with this condition such as advanced age, anticoagulation, arteriosclerosis, hypertension, coughing, pregnancy, renal disease, intensive rectus muscle contractions, hemostatic disorders, abdominal surgery and/or abdominal wall injections (6). However, the most frequent predisposing factor is anticoagulation therapy and the most inducible factor is coughing (7). Clinical manifestations include abdominal pain, abdominal wall ecchymosis, drop in hematocrit, and a positive Carnett's sign (increase in abdominal pain when a supine patient tenses his or her abdominal wall by lifting their head and shoulders off the examination table, indicating the abdominal wall and not the abdominal cavity as the source of pain) (8).

\subsection{Conclusion}

Anterior abdominal wall hematoma should be considered in the differential diagnosis of acute abdominal pain among elderly patients, especially those who are under anti-coagulation therapy. Conservative treatment is conceivable in most cases. Early diagnosis is compulsory to avoid morbidity or unnecessary surgery. This patient is managed conservatively and discharged from the hospital three days later.

\section{Acknowledgments}

None to declare.

\section{Footnotes}

Authors' Contribution: Ahmad Hormati was the responsible physician; Pezhman Alavinejad was the writer of the manuscript.

Conflict of Interest: The authors had no conflict of interest. 


\section{References}

1. Bohner H, Yang Q, Franke C, Verreet PR, Ohmann C. Simple data from history and physical examination help to exclude bowel obstruction and to avoid radiographic studies in patients with acute abdominal pain. Eur J Surg. 1998;164(10):777-84. doi: 10.1080/110241598750005435. [PubMed: 9840308].

2. Stone R. Acute abdominal pain. Lippincott's primary care practice. 1997;2(4):341-57.

3. Cherry WB, Mueller PS. Rectus sheath hematoma: review of 126 cases at a single institution. Medicine (Baltimore). 2006;85(2):105-10. doi: 10.1097/01.md.0000216818.13067.5a. [PubMed:16609349].

4. Nourbakhsh E, Anvari R, Nugent K. Abdominal wall hematomas associated with low-molecular-weight heparins: an important complication in older adults. J Am Geriatr Soc. 2011;59(8):1543-5. doi: 10.1111/j.15325415.2011.03529.x. [PubMed: 21848819].
5. Kaw D, Malhotra D, editors. Hematology: issues in the dialysis patient: platelet dysfunction and end-stage renal disease. Seminars in dialysis. 2006; Wiley Online Library; pp. 317-22.

6. Fitzgerald JE, Fitzgerald LA, Anderson FE, Acheson AG. The changing nature of rectus sheath haematoma: case series and literature review. Int J Surg. 2009;7(2):150-4. doi: 10.1016/j.ijsu.2009.01.007. [PubMed: 19261556].

7. Berna JD, Zuazu I, Madrigal M, Garcia-Medina V, Fernandez C, Guirado F. Conservative treatment of large rectus sheath hematoma in patients undergoing anticoagulant therapy. Abdom Imaging. 2000;25(3):230-4. [PubMed: 10823439].

8. Donaldson J, Knowles $\mathrm{CH}$, Clark SK, Renfrew I, Lobo MD. Rectus sheath haematoma associated with low molecular weight heparin: a case series. Ann R Coll Surg Engl. 2007;89(3):309-12. doi: 10.1308/003588407X179152. [PubMed: 17394721]. 